\title{
Oral appliance in sleep apnea treatment : respiratory and clinical effects and long-term adherence
}

\section{Bachour, Patrick}

2016-05

Bachour , P , Bachour , A , Kauppi , P , Maasilta , P , Makitie , A \& Palotie , T 2016 , ' Oral appliance in sleep apnea treatment : respiratory and clinical effects and long-term adherence ' , Sleep and Breathing , vol. 20 , no. 2 , pp. 805-812 . https://doi.org/10.1007/s11325-015-1301-0

http://hdl.handle.net/10138/223987

https://doi.org/10.1007/s11325-015-1301-0

publishedVersion

Downloaded from Helda, University of Helsinki institutional repository.

This is an electronic reprint of the original article.

This reprint may differ from the original in pagination and typographic detail.

Please cite the original version. 


\title{
Oral appliance in sleep apnea treatment: respiratory and clinical effects and long-term adherence
}

\author{
Patrick Bachour $^{1}$ • Adel Bachour ${ }^{1}$ - Paula Kauppi ${ }^{2}$ - Paula Maasilta ${ }^{1}$ - Antti Mäkitie ${ }^{3,4}$. \\ Tuula Palotie 5
}

Received: 2 September 2015 /Revised: 14 December 2015 / Accepted: 21 December 2015 /Published online: 11 January 2016

(C) Springer-Verlag Berlin Heidelberg 2016

\begin{abstract}
Purpose There is an increasing tendency to use oral appliance (OA) as an alternative treatment for sleep apnea. Here we report the long-term adherence and clinical effects of OA therapy.

Methods All sleep apnea patients treated at the Department of Dentistry between the years 2006 and $2013(n=1208)$ were reviewed. A questionnaire about $\mathrm{OA}$ adherence, asthma symptoms (Asthma Control Test ${ }^{\mathrm{TM}}, \mathrm{ACT}$ ), and general health was sent to all patients who continued OA therapy after the 1 -month follow-up visit $(n=811)$. OA was adjusted to obtain at least $70 \%$ of the maximal protrusion of the mandible. Results The response rate was 37.4\% (99 women, 204 men). The mean \pm SD age and BMI were $58.7 \pm 10.3$ years and $27.3 \pm 4.0 \mathrm{~kg} / \mathrm{m}^{2}$, respectively. During the mean follow-up period of 3.3 years, there was no significant variation in BMI.
\end{abstract}

Patrick Bachour

patrick.bachour@helsinki.fi

$1 \quad$ Heart and Lung Center, Sleep Unit, University of Helsinki and Helsinki University Hospital, P.O.BOX 160, FI-00029 HUS Helsinki, Finland

2 Inflammation Center, Allergy Department, University of Helsinki and Helsinki University Hospital, P.O.BOX 160, FI-00029 HUS Helsinki, Finland

3 Department of Otorhinolaryngology - Head and Neck Surgery, University of Helsinki and Helsinki University Hospital, P.O.BOX 160, FI-00029 HUS Helsinki, Finland

4 Division of Ear, Nose and Throat Diseases, Department of Clinical Sciences, Intervention and Technology, Karolinska Institutet, Stockholm, Sweden

5 Department of Oral and Maxillofacial Diseases, University of Helsinki, Helsinki, Finland
Forty-one patients abandoned OA therapy yielding an adherence rate of $86 \%$. Ninety-seven percent of patients used OA $\geq 4 \mathrm{~h}$ /day, and the mean daily use was $7.2 \pm 1.1 \mathrm{~h}$. The ACT score improved with OA use from $16.0 \pm 5.9$ to $20.1 \pm 3.8$ ( $p=0.004)$, indicating better asthma control. The apnea and hypopnea index decreased significantly from $27 \pm 19$ at baseline to $10 \pm 10$ with OA therapy $(p=0.001)$.

Conclusions After a 1-month trial period, the long-term adherence to oral appliance was good. OA therapy decreased apneas and hypopneas significantly, and its longterm use was associated with an improvement in respiratory and asthma symptoms.

Keywords Sleep study · Asthma - Questionnaire · Oral sleep apnea appliance

\section{Introduction}

Obstructive sleep apnea (OSA) is a sleep disorder in which the airways collapse or become partially or completely blocked during sleep, resulting in breathing difficulties and pauses [1]. OSA symptoms include snoring, pauses in breathing, daytime sleepiness, nocturia, and morning headaches. Untreated OSA can have negative cardiovascular effects such as hypertension and arrhythmia [2].

OSA can be treated effectively with continuous positive airway pressure (CPAP) which is the first-line therapy. CPAP forces the airways to remain open, reducing the pauses in breathing and relieving sleep apnea symptoms. The downside of CPAP is poor adherence; about one third of patients have dropped out of treatment after 1 year [3, 4].

An oral sleep apnea appliance (OA) that reduces OSA symptoms by advancing the mandible and increasing oropharyngeal space is a viable alternative in the treatment of OSA 
for patients who are unable or unwilling to use CPAP [5-7]. The OA therapy has been reported to improve arterial stiffness, glucose metabolism, and insulin resistance in mild to moderate OSA patients after 1 year of treatment [8]. In addition, the OA has another therapeutic effect as a bite splint for bruxism [9]. Moreover, the American academy of dental sleep medicine recently recommended that sleep physicians should consider prescription of oral appliances, rather than no treatment, for adult patients with obstructive sleep apnea who are intolerant of CPAP therapy or prefer alternate therapy [10]. In a randomized placebo-controlled study, Marklund et al. showed that a custom-made oral appliance reduces snoring, obstructive sleep apnea, and restless legs without effects on daytime sleepiness and quality of life when compared with a control group [11]. Similar to CPAP therapy, the adherence to OA therapy is also poor $[12,13]$. Patients with short maxilla and mandible have been reported to have better adherence to OA [13].

Earlier, we had observed (data not shown) a reduction in respiratory and asthma symptoms with long-term CPAP therapy in adult patients suffering from OSA and asthma. It is still unclear whether the amelioration in asthma symptoms was a direct effect of CPAP on the airways (also the small airways) or due to the proper treatment of OSA. Therefore, a question arises whether other sleep apnea treatments, such as OA, have similar effects on asthma symptoms.

The aim of this study was to assess through questionnaires the quality of life and respiratory and asthma symptoms in a series of patients using oral appliance for OSA treatment.

\section{Methods}

We reviewed the hospital charts of all patients who had been treated at the Departments of Oral and Maxillofacial Diseases, $\mathrm{HUCH}$, Helsinki, Finland, for a diagnosis of sleep apnea (G47.3) between the years 2006 and 2013.

Of the 1208 patients reviewed, we selected 811 whose oral appliance therapy had continued after their first follow-up visit at 1 month. We sent a questionnaire to all these patients, which included the Finnish version of the Asthma Control Test ${ }^{\mathrm{TM}}$, and questions of the use of oral appliance, CPAP therapy state, BMI, comorbidity, medications, and modifications in sleep apnea symptoms (Fig. 1).

We reviewed randomly half of the files from the nonanswering group for general characteristics in order to evaluate the representativeness of the responding group.

Asthma was defined as a self-reported physician-diagnosed disease and a special reimbursement for asthma medication by the Social Insurance Institution. Asthma severity was measured with a visual analogue scale (VAS) where 0 meant no symptoms and 100 meant severe symptoms [14], and with the
Finnish version of the Asthma Control Test ${ }^{\mathrm{TM}}$ (ACT score range from 5 to 25).

We asked the following question: Did you use an occlusal splint for teeth grinding before your oral appliance? If yes, please describe how the oral appliance has relieved your teeth grinding symptoms (abolished, significant improvement, mild improvement, no efficacy).

The questions related to nasal medications comprised modifications in the use of nasal decongestants, steroids, and moisturizers with long-term use of OA. The responses were classified in four categories (stopped, reduced, no change, or increased).

Oral appliance therapy was indicated as a therapy for OSA in patients who refused CPAP therapy or in those whose CPAP therapy failed. All patients should have an apnea hypopnea index $(\mathrm{AHI}) \geq 15 / \mathrm{h}$ or an $\mathrm{AHI} \geq 5 / \mathrm{h}$ along with considerable daytime sleepiness that manifested and interfered with daily life routines. A BMI over 35 was considered as a relative contraindication to OA therapy. We used two types of appliances, Herbst and Herner (Fig. 2). Both have two acrylic splints and two beams. All our oral appliances were done by our experienced technician with high level of construction consistency.

A dentist specialized in oral appliance manually adjusted the device for each patient to obtain $70 \%$ of the maximal protrusion of the mandible. The amount of vertical opening was one to $2 \mathrm{~mm}$ in the incisor area and $3 \mathrm{~mm}$ in the molar area. The first follow-up visit was planned 1 month later. The protrusion was then increased by $1-3 \mathrm{~mm}$ if the patient still reported snoring or witnessed apnea. No further adjustments were performed.

\section{Statistical analyses}

A software statistical package (IBM SPSS ${ }^{\circledR}$ Statistics 22.0, Armonk, NY, USA) was used to analyze the data. The Student's $t$ test or chi-square test was used to evaluate the significance of modifications caused by oral appliance treatment for parametric and non-parametric data accordingly. A $p$ value of $<0.05$ was considered a statistically significant difference. Missing data were excluded from analysis. Only patients who used OA as their only therapy for OSA were considered in the analysis; we therefore excluded all patients who abandoned OA therapy or returned partially to CPAP therapy.

\section{Results}

We received 303 completed replies from the 811 questionnaires sent, yielding a response rate of $37.4 \%$. No difference in gender, baseline AHI, or native language (Finnish or Swedish) was found between the responders and non-responders. The 
Fig. 1 Flowchart of patient selection

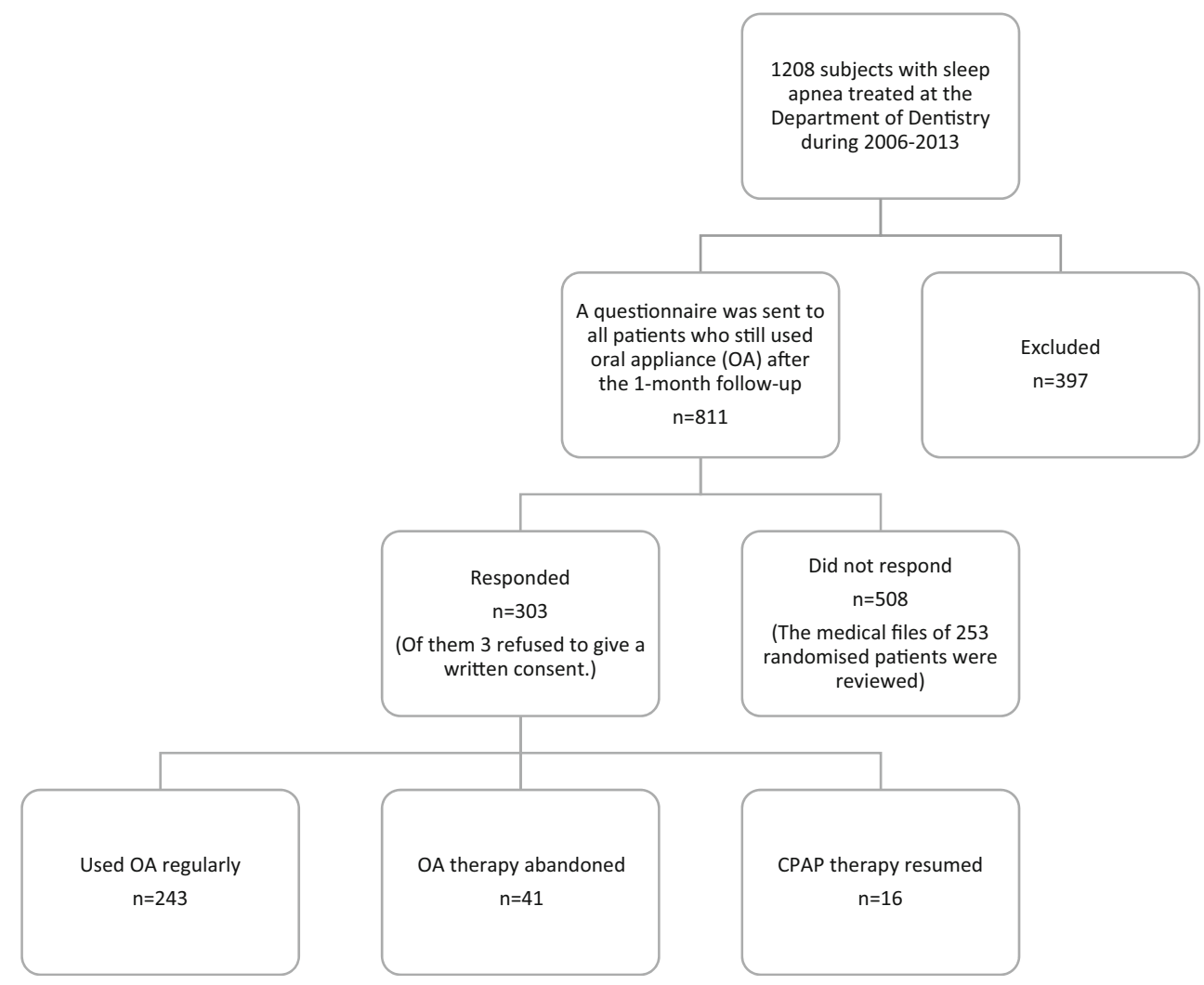

percentage of patients who abandoned OA therapy or resumed CPAP therapy was not significantly $(p=0.458)$ different between responders and non-responders (19.0 and $21.7 \%$, respectively). However, the respondents were older, were less obese, and had started OA therapy more recently compared with the non-respondents (59.3 vs. 54.4 years, $p=0.001 ; 27.1$ vs. $28.2 \mathrm{~kg} / \mathrm{m}^{2}, p=0.005 ; 3.3$ vs. 3.7 years, $p=0.024$, respectively, Table 1 ).

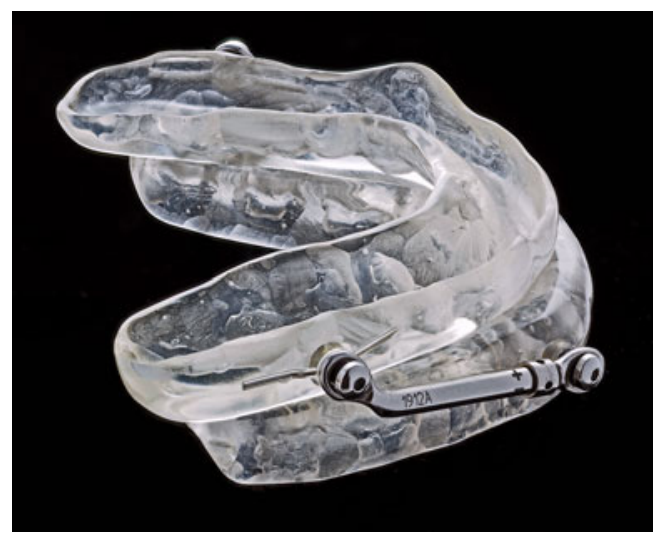

Fig. 2 Herner type oral appliance. The device consists of two acrylic splints and two beams. Please notice the protrusion of the mandible

\section{Patient demographics and oral appliance use}

Of the 300 respondents, 243 patients (81\%) were still using $\mathrm{OA}$, and 41 (14\%) had abandoned and $16(5 \%)$ had resumed occasional CPAP therapy along with OA therapy. During the mean follow-up period of 3.3 years, the adherence to OA therapy was $86 \%$. The mean OA daily use data were not available for two patients; otherwise, the patients used their $\mathrm{OA}$ for a mean of $7.17 \mathrm{~h}$ a day $(\mathrm{SD} \pm 1.11)$. The daily $\mathrm{OA}$ use was below $4 \mathrm{~h}$ for only seven patients (3\%), yielding a good daily adherence rate of $97 \%$ (Fig. 3). The baseline AHI values

Table 1 Characteristics of 811 patients with oral appliance therapy

\begin{tabular}{llll}
\hline & Answered & Non-answered & $p$ \\
\hline Number & 303 & 508 & \\
Female (\%) & 33 & 27 & 0.093 \\
BMI baseline $\left(\mathrm{kg} / \mathrm{m}^{2}\right)$ & 27.1 & 28.2 & 0.005 \\
Age (years) & 59.3 & 54.4 & 0.001 \\
AHI, all respondents & 22.2 & 24.1 & 0.171 \\
Swedish speaking (\%) & $7 \%$ & $5 \%$ & 0.112 \\
OA since (years) & 3.3 & 3.7 & 0.024 \\
OA stopped & $19.0 \%$ & $21.7 \%$ & 0.458 \\
\hline
\end{tabular}

$O A$ oral appliance 
Fig. 3 Distribution of the reported long-term daily oral appliance use

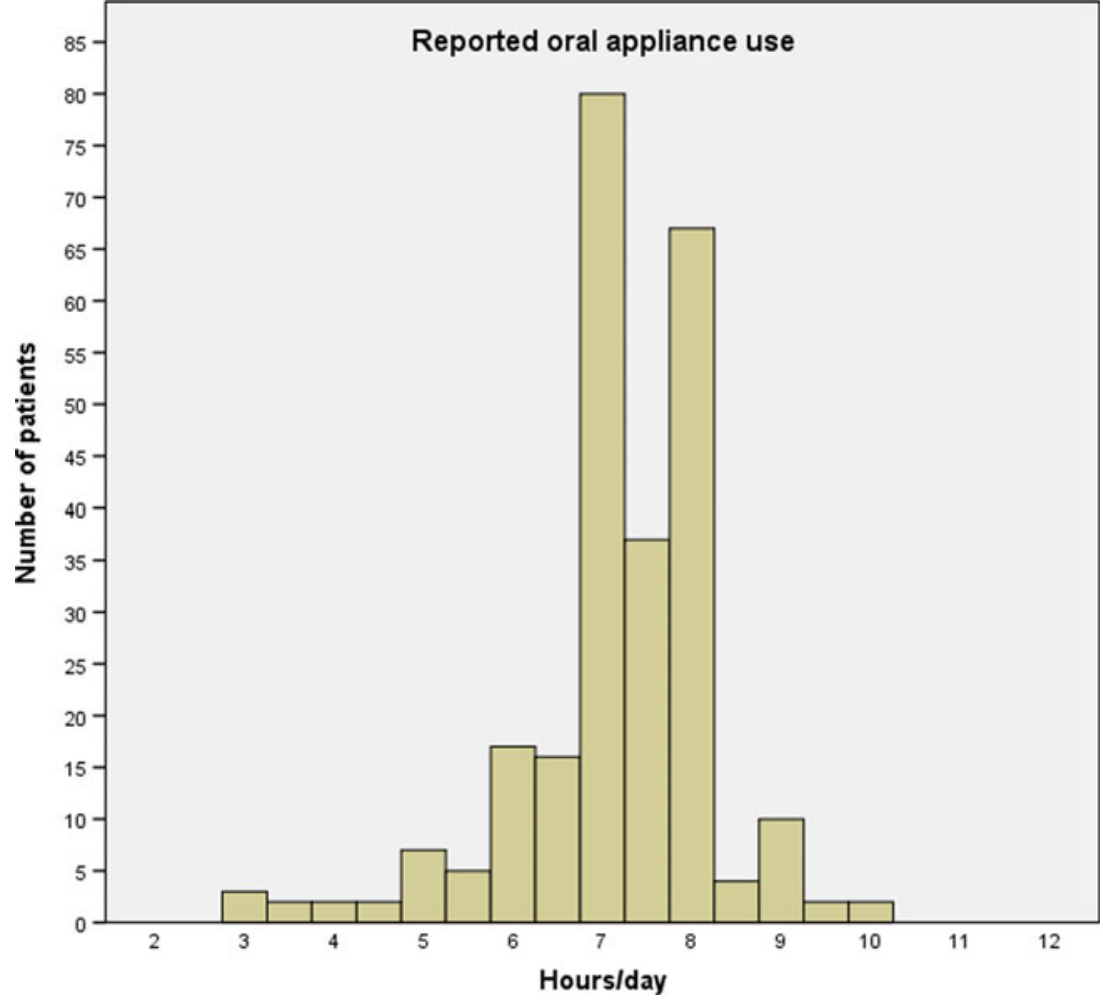

were not available for 52 patients. For 12 patients, the diagnosis of OSA was written in the patient's file, although their AHI values were below $5 / \mathrm{h}$. Baseline AHI was $\geq 30$ in 59 patients, between 15 and 30 in 96 patients, and between 5 and 15 in 81 patients. The results of sleep studies with OA were available in 49 patients. We noticed a significant $(p=0.001)$ reduction in AHI values with OA therapy, $27 \pm 19$ versus $10 \pm 10$, respectively. We included in the analysis these 243 patients who used OA as their only treatment for OSA. Their mean (SD) age was 58.7 (10.3) years, 84 patients were female $(34.6 \%)$, and 34 (13.9\%) were active smokers. The patients did not report a significant modification in their BMI with long-term OA therapy $\left(27.3 \mathrm{~kg} / \mathrm{m}^{2}\right.$ baseline, $27.3 \mathrm{~kg} / \mathrm{m}^{2}$ with $\left.\mathrm{OA}, p=0.625\right)$; the objectively measured baseline BMI $\left(27.2 \mathrm{~kg} / \mathrm{m}^{2}\right)$ showed the same results as the self-reported ones $(p=0.763)$.

\section{Asthma}

A total of 18 patients fulfilled the criteria of asthma yielding a prevalence of $7.4 \%$ for asthma among long-term OA users. The patients rated their asthma control better with OA treatment since the mean score of the Asthma Control Test ${ }^{\mathrm{TM}}$ (ACT) improved with long-term OA therapy from 16.0 \pm 5.9 to $20.1 \pm 3.8$ ( $p=0.004$; Fig. 4). In addition, the use of VAS for self-evaluating asthma symptoms showed a tendency for improvement, as the VAS score decreased from $50.9(\mathrm{SD} \pm 29.6)$ to $43.2(\mathrm{SD} \pm 24.4)$ but without reaching statistical significance $(p=0.123)$. Asthma symptoms improved most with OA therapy in patients whose asthma

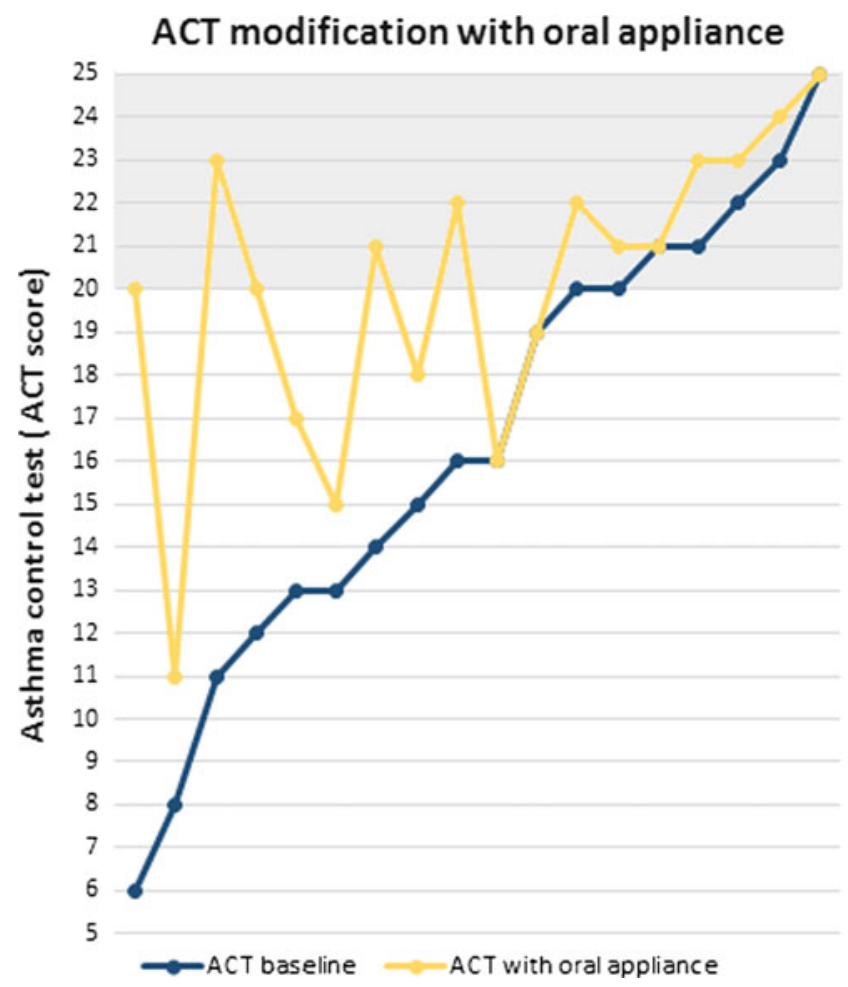

Fig. 4 The effect of long-term oral appliance therapy for sleep apnea on Asthma Control Test ${ }^{\mathrm{TM}}$ (ACT) in asthmatics. An ACT score $\geq 20$ indicates good asthma control (gray area) 
was not under control (scored 19 or less points in the ACT) before OA therapy. However, even patients whose asthma was controlled (scored 20 or more points in the ACT) showed some improvement (Fig. 4).

\section{Quality of life and other modifications}

With long-term OA therapy, $83 \%$ (197 patients) reported an improvement in their overall quality of life, $15 \%$ reported no changes, and the quality of life worsened for $2 \%$. Scores of the self-reported ability to work improved in 121 (67\%) patients, stayed unchanged in $31 \%$, and worsened in $2 \%$ of patients. The mood of patients improved in $64 \%$, stayed unchanged in $33 \%$, and decreased in $3 \%$. Moreover, symptoms of headaches showed the same tendency, where $34 \%$ of patients reported a decrease in symptoms, $58 \%$ reported no headaches or no modifications in their headache symptoms, and $8 \%$ reported worsened headaches (Fig. 5). The improvements were not related to gender.

We noticed a significant decrease in the frequency of nocturia from 1.24 times/night at baseline to 0.96 times/ night with long-term OA therapy ( $p=0.000, N=232$ ). Significant improvement was noticed in both men and women, separately.

A total of 41 (17\%) patients had reported using hypnotics regularly or occasionally. The majority ( $73 \%$ ) of them showed no modification in the consumption of hypnotics with the long-term use of OA, only $17 \%$ managed to reduce their consumption, and for $10 \%$ the consumption was increased.

\section{Users of occlusal splint}

A total of 29 (12\%) patients had used an occlusal splint. Their occlusal splint was replaced by an OA as the treatment for OSA. Effectively, the application of OA was also associated with an abolishment in teeth-grinding-related symptoms in $39 \%$ of patients, a significant improvement in $36 \%$, a mild improvement in $21 \%$, and only one patient ( $4 \%$ ) reported no efficacy.

\section{Nasal medication}

The long-term OA therapy was associated with discontinuation of use of nasal decongestants in $37 \%$ of users, a reduction of use in $19 \%$, no change in $41 \%$, and an increase in $3 \%$. Similar results were observed with nasal steroids, where use stopped in $34 \%$ of users, decreased in $18 \%$, remained unchanged in $45 \%$, and increased in $3 \%$. Moisturizing nasal spray use stopped in $27 \%$ of users, decreased in $18 \%$, remained unchanged in $49 \%$, and increased in $6 \%$ (Fig. 6).

\section{Discussion}

In this study, we sent a questionnaire to 811 patients treated with an oral appliance (OA) for OSA. We aimed at estimating the effectiveness of sleep apnea therapy by OA on asthmaand sleep-apnea-related symptoms. OA had reduced the AHI significantly. A tendency of improvement in respiratory and
Fig. 5 Benefits and side effects of oral appliance therapy for sleep apnea

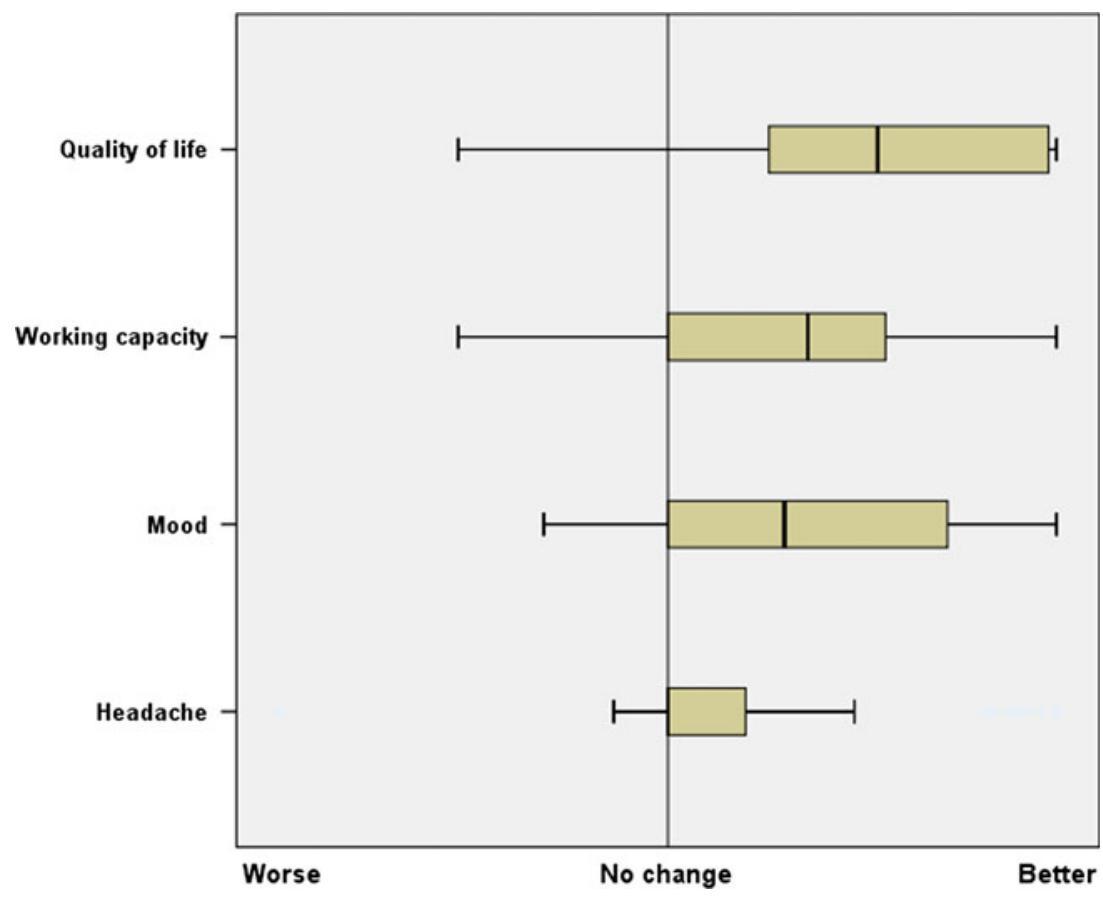


Fig. 6 Changes in the use of nasal medications with long-term oral appliance as a therapy for sleep apnea

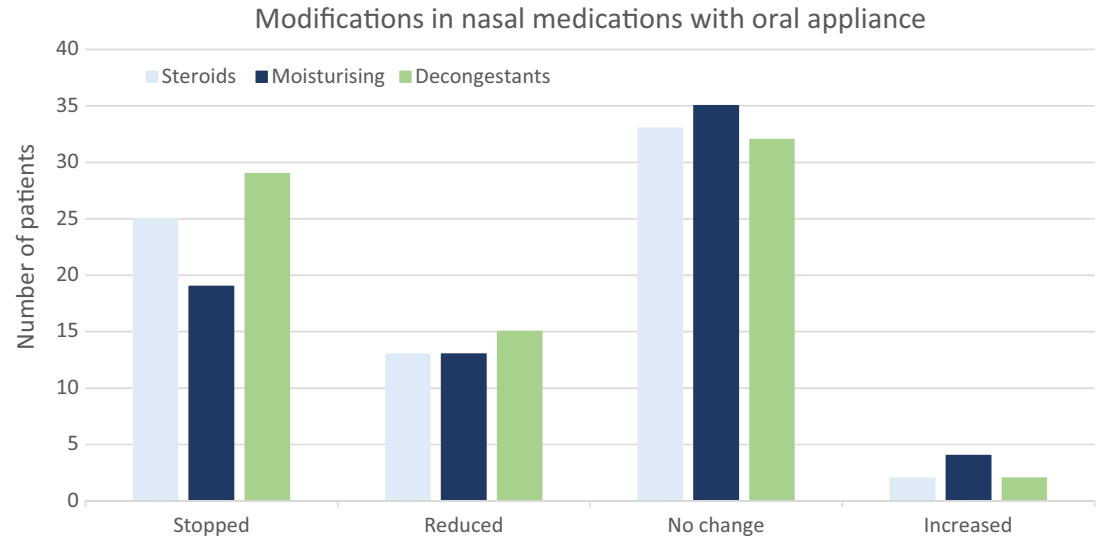

asthma symptoms with the long-term OA therapy was observed. There was also good long-term adherence to OA therapy in those patients who still used their appliance after their first follow-up visit at 1 month.

The pathophysiology of asthma improvement, in asthmatic patients receiving specific treatment for sleep apnea, is not fully understood. Coexisting asthma and sleep apnea have a synergistic effect on each other, and multiple pathophysiologic theories on OSA effects on asthma have been proposed [15]. Snoring and reduced airflow in OSA increase the mechanical stress on the lower airways, and the closure of upper airways in OSA could trigger bronchoconstriction through vagal reflexes. Other theories include the effects of upper airway inflammation, hypoxia, vascular endothelial growth factor (VEGF) and leptin levels on the lower airways, and asthma severity [15]. Stabilization of the airways by CPAP therapy could, in part, explain the amelioration of respiratory and asthma symptoms, although this needs to be confirmed. The tendency of improvement in respiratory and asthma symptoms with long-term OA therapy, reported in this study, suggests that other pathophysiological mechanisms are also involved. Interestingly, no patients had any negative impacts on asthma symptoms with long-term oral appliance therapy measured with the ACT.

The adherence to OA therapy varies between a range of 4 to $76 \%$ at the end of 1 year [12]. Effectively, the adherence depends on the balance between the perception of benefit and side effects. In our previous study on 96 patients, we reported an OA adherence rate of $56 \%$ at 6 months [13]. In this study, we noticed that once the patient had accepted the OA after 1 month of use, the adherence thereafter was relatively high at $86 \%$ after 3.3 years and only $14 \%$ abandoned therapy during the 3.3-year follow-up. Therefore, the vast majority of OA therapy dropouts are witnessed during the first 1 month. Consequently, we recommend special attention during that period. In addition to the high adherence rate reported in this study, the minimum recommended daily use ( $>4 \mathrm{~h})$ [10] was achieved in $97 \%$ of patients. The high daily use could be the consequence of our strict OA indication policy, as we do not start OA therapy, at our university hospital, in simple snorers or as a backup or supplementary therapy to CPAP. Despite these restrictions, we noticed that both OA and CPAP therapies were still available in 16 of our patients, yet we suspect that their CPAP use stayed marginal, as their daily OA use was relatively high.

About $12 \%$ of our patients used to have an occlusal splint for teeth grinding. They reported that the OA has also helped them in relieving their tooth-grinding-related symptoms. Our findings showed similar results as those reported recently by Carra et al. on an adolescent population suffering from snoring and headaches. They noticed an amelioration of bruxismrelated symptoms with the short-term use of OA [9]. Effectively, we cannot confirm the diagnosis of bruxism in our previous occlusal splint users, neither could we describe what specific symptoms were relieved by the application of OA. It is worth mentioning that all of the occlusal splints were prescribed by authorized dentists. It has been reported that the effectiveness of mandibular advancing OA on bruxism symptoms is equal to or even better than the effectiveness of occlusal splint, but the latter is the first line of treatment due to the possible adverse effects of OA use [16]. Patients suffering from both bruxism and mild OSA may be candidates for first-line OA therapy for their OSA, and this option should be investigated.

Recently, the use of CPAP therapy was reported to promote an increase in the body mass index [17]. Interestingly, the BMI of our patients stayed unchanged with the long-term use of OA. Our patients at the baseline were not considerably obese, as we considered a BMI over $35 \mathrm{~kg} / \mathrm{m}^{2}$ as a relative contraindication to OA therapy.

Our patients reported a decrease in their use of nasal medications with long-term OA therapy. This finding follows the same trend that we noticed for respiratory and asthma symptoms. We do not have a rational explanation for this reduction in nasal medication; however, we hypothesize that the restoration of nasal breathing with the reduction of apneas make the airflow more physiological and increases the well-being of the nasal mucosa, reducing chronic dryness and swelling. We 
need more studies to confirm our finding and to explain the mechanism of this improvement. Anyway, a reduction in the consumption of medications is always welcomed, leading also to a reduction in total health care costs.

Marklund et al. recently reported that OA therapy for OSA produced no changes in quality of life. We showed a tendency of improvement in both quality of life and in working capacity. Effectively, our patients had more severe sleep apnea, and our results reflect a long-term therapy effect; meanwhile, their patients had mild to moderate sleep apnea and the follow-up period was 4 months.

Franco et al. [18] treated patients suffering from morning headache and orofacial pain with a similar OA to the one used in this study and concluded that the short-term use of OA was associated with significant reductions in morning headaches. Their patients did not suffer from sleep-disordered breathing. Moreover, they used the OA in both neutral and advanced positions. They suggest that the effectiveness of OA in reducing morning headaches may be linked to the reduction in rhythmic masticatory muscle activity without relation to sleep apnea. We applied the OA in the advanced position and all our patients suffered from sleep apnea, yet our results are in concordance with theirs, as we noticed a reduction in headache symptoms. However, sleep disorders have been linked to risk factors for morning headaches [19], and thus, treating sleep apnea may improve headaches [20].

Our study has some limitations. As we used a questionnaire, with no control group available, we could not draw solid conclusions about the efficacy of the OA. The results were measured based on the subjective reports of the patients, and recall bias in reporting the symptoms cannot be totally ruled out. Furthermore, the number of patients with asthma was small. We did not use an integrated miniature micro-recorder to measure the adherence objectively, as this technology was made available only recently [21]. The response rate of this was study was not high; however, the studied group represented the whole population fairly well. In spite of the abovementioned restrictions, this study covered all the patients with OA therapy at our institution for a period of 7 years. Finally, our asthma diagnosis followed the strict national guidelines excluding possibilities of diagnosis aberrations.

The results of this observational cohort study warrant future studies to evaluate OA treatment effectiveness on asthma symptoms.

\section{Conclusion}

After the 1-month oral appliance trial period, the adherence to the treatment was good. A tendency of improvement in respiratory and asthma symptoms was observed with long-term OA therapy for sleep apnea. Oral appliance treatment was also associated with improvements in mood, quality of life, headache, working capacity, and nocturia.

\section{Compliance with ethical standards}

Conflict of interest Paula Kauppi is employed by HUS, Skin and Allergy Hospital as the Chief Specialist of the Adult Unit of the Department of Allergy and is a member of ERS. She has been a consultant for Leiras-Takeda Pharmaceuticals, GSK, and BoehringerIngelheim for the past 3 years.

All other authors certify that they have no affiliations with or involvement in any organization or entity with any financial or non-financial interest in the subject matter or materials discussed in this manuscript. This study was funded by the Helsinki University Hospital Research Fund (V1016SK001).

Ethical consideration An institutional research approval at the Helsinki University Hospital, Department of Internal Medicine, was granted for the study (code $\S 10211.6 .2014$, permit number 202/13/03/02/2014). Written consent was requested from each patient.

\section{References}

1. Dempsey JA, Veasey SC, Morgan BJ, O’Donnell CP (2010) Pathophysiology of sleep apnea. Physiol Rev 90:47-112

2. Kendzerska T, Mollayeva T, Gershon AS, Leung RS, Hawker G, Tomlinson G (2014) Untreated obstructive sleep apnea and the risk for serious long-term adverse outcomes: a systematic review. Sleep Med Rev 18:49-59

3. Kreivi HR, Maasilta P, Bachour A (2014) Willingness score obtained after a short CPAP trial predicts CPAP use at 1 year. Sleep Breath 18:207-213

4. Sawyer AM, Gooneratne NS, Marcus CL, Ofer D, Richards KC, Weaver TE (2011) A systematic review of CPAP adherence across age groups: clinical and empiric insights for developing CPAP adherence interventions. Sleep Med Rev 15:343-356

5. Doff MH, Hoekema A, Wijkstra PJ, van der Hoeven JH, Huddleston Slater JJ, de Bont LG, Stegenga B (2013) Oral appliance versus continuous positive airway pressure in obstructive sleep apnea syndrome: a 2-year follow-up. Sleep 36:1289-1296. doi:10.5665/sleep.2948

6. Hoekema A (2006) Efficacy and comorbidity of oral appliances in the treatment of obstructive sleep apnea-hypopnea: a systematic review and preliminary results of a randomized trial. Sleep Breath 10:102-103

7. Marklund M, Franklin KA (2015) Treatment of elderly patients with snoring and obstructive sleep apnea using a mandibular advancement device. Sleep Breath 19:403-405

8. Galic T, Bozic J, Ivkovic N, Gunjaca G, Ticinovic T, Dogas Z (2015) Effects of mandibular advancement device treatment on arterial stiffness and glucose metabolism in patients with mild to moderate obstructive sleep apnea: a prospective 1 year study. Sleep Breath. doi:10.1007/s11325-015-1186-y

9. Carra MC, Huynh NT, El-Khatib H, Remise C, Lavigne GJ (2013) Sleep bruxism, snoring, and headaches in adolescents: short-term effects of a mandibular advancement appliance. Sleep Med 14: 656-661

10. Ramar K, Dort LC, Katz SG, Lettieri CJ, Harrod CG, Thomas SM, Chervin RD (2015) Clinical practice guideline for the treatment of obstructive sleep apnea and snoring with oral appliance therapy: an update for 2015. J Clin Sleep Med 11:773-827 
11. Marklund M, Carlberg B, Forsgren L, Olsson T, Stenlund H, Franklin KA (2015) Oral appliance therapy in patients with daytime sleepiness and snoring or mild to moderate sleep apnea a randomized clinical trial. JAMA Intern Med 175(8):1278-1285

12. Hoffstein V (2007) Review of oral appliances for treatment of sleep-disordered breathing. Sleep Breath 11(1):1-22

13. Ingman T, Arte S, Bachour A, Bäck L, Mäkitie A (2013) Predicting compliance for mandible advancement splint therapy in 96 obstructive sleep apnea patients. Eur J Orthod 35:752-757

14. Sullivan PW, Smith KL, Ghushchyan VH, Globe DR, Lin SL, Globe G (2013) Asthma in USA: its impact on health-related quality of life. J Asthma 50:891-899

15. Puthalapattu S, Ioachimescu OC (2014) Asthma and obstructive sleep apnea: clinical and pathogenic interactions. J Investig Med 62(4):665-675

16. Huynh N, Manzini C, Rompré PH, Lavigne GJ (2007) Weighing the potential effectiveness of various treatments for sleep bruxism. $\mathrm{J}$ Can Dent Assoc 73:727-730
17. Drager LF, Brunoni AR, Jenner R, Lorenzi-Filho G, Benseñor IM, Lotufo PA (2015) Effects of CPAP on body weight in patients with obstructive sleep apnoea: a meta-analysis of randomised trials. Thorax 70(3):258-264

18. Franco L, Rompre PH, de Grandmont P, Abe S, Lavigne GJ (2011) A mandibular advancement appliance reduces pain and rhythmic masticatory muscle activity in patients with morning headache. J Orofac Pain 25:240-249, Summer

19. Rains JC (2008) Chronic headache and potentially modifiable risk factors: screening and behavioral management of sleep disorders. Headache 48(1):32-39

20. Johnson KG, Ziemba AM, Garb JL (2013) Improvement in headaches with continuous positive airway pressure for obstructive sleep apnea: a retrospective analysis. Headache 53:333-343

21. Vanderveken OM, Dieltjens M, Wouters K, De Backer WA, Van de Heyning PH, Braem MJ (2013) Objective measurement of compliance during oral appliance therapy for sleep-disordered breathing. Thorax 68:91-96 